\title{
Efeito de extratos de plantas com atividade inseticida no controle de Microtheca ochroloma Stal (Col.: Chrysomelidae), em laboratório
}

\author{
Sônia Thereza Bastos Dequech ${ }^{1 *}$ \\ Carla Daniele Sausen ${ }^{2}$ \\ Cíntia Grendene Lima ${ }^{2}$ \\ Rafael Egewarth ${ }^{2}$ \\ ${ }^{1}$ Universidade Federal de Santa Maria (UFSM), Centro de Ciências Rurais \\ Departamento de Defesa Fitossanitária, CEP 97105-900, Santa Maria - RS, Brasil \\ ${ }^{2}$ Curso de Agronomia, UFSM, Santa Maria, RS, Brasil \\ *Autor para correspondência \\ soniabd@terra.com.br
}

\section{Resumo}

Extratos de plantas com atividade inseticida foram testados no controle de Microtheca ochroloma (Col.: Chrysomelidae), uma importante praga de brassicáceas nas fases larval e adulta. Duas larvas com três dias de idade, mantidas sob condições de laboratório (T:25 ${ }^{\circ} \mathrm{C}$, U.R:70\% e 14 horas de fotofase), foram colocadas em tubos de vidro contendo folha de couve-chinesa (Brassica chinensis) previamente tratada com extratos aquosos $(10 \% \mathrm{p} / \mathrm{v})$ de folha de cinamomo (Melia azedarach), de ramo de cinamomo e de pó-de-fumo (Nicotiana tabacum). O mesmo procedimento foi repetido em dois ensaios com insetos adultos. No primeiro, foram utilizados todos os extratos anteriormente citados mais o extrato de DalNeem (produto comercial à base de Azadirachta indica). No segundo, os insetos foram expostos a extratos de frutos de pimenta malagueta (Capsicum frutescens) e de folhas de pitangueira (Eugenia uniflora), de jambolão (Syzygium cuminii) e de eucalipto (Eucalyptus sp.). Todos os testes constaram de 10 insetos por tratamento, com cinco repetições no primeiro ensaio com insetos adultos e com seis repetições nos demais. As observações foram realizadas diariamente até o quinto dia, objetivando avaliar a mortalidade dos insetos. Todos os extratos testados resultaram num controle efetivo de larvas de M. ochroloma. Em relação aos insetos adultos, apenas os extratos de pó-de-fumo e de DalNeem apresentaram eficiência de controle.

Unitermos: extratos vegetais, plantas inseticidas, Melia azedarach

\section{Abstract}

Effect of extracts of plants with insecticidal activity on the control of Microtheca ochroloma Stal (Col: Chrysomelidae) in the laboratory. Extracts of plants with insecticidal activity were tested on the control of Microtheca ochroloma (Col.: Chrysomelidae), an important insect-pest of Brassicaceae, in the larval and adult phases. Two 3 -day-old larvae, kept under laboratory conditions $\left(25^{\circ} \mathrm{C}\right.$ temperature, $70 \%$ relative humidity and 14 hours of photophase), were placed in a glass tube with a leaf of Chinese cabbage (Brassica chinensis) previously treated with aqueous extracts $(10 \% \mathrm{p} / \mathrm{v})$ of chinaberry leaf (Melia azedarach), chinaberry branch, 
and tobacco powder (Nicotiana tabacum). The same procedure was repeated in two assays with adult insects. In the first assay, all the previously-mentioned extracts were used, in addition to DalNeem (commercial product of Azadirachta indica). In the second, the insects were exposed to extracts of tabasco pepper fruits (Capsicum frutescens), Surinam cherry (Eugenia uniflora), jambolan (Syzygium cuminii) and eucalyptus leaves (Eucalyptus $\mathrm{sp.).} \mathrm{All} \mathrm{the} \mathrm{tests} \mathrm{consisted} \mathrm{of} 10$ insects per treatment, with five repetitions in the first test using adult insects and six repetitions in the others. Observations were made daily up to the fifth day, aiming to evaluate the mortality of the insects. All the tested extracts resulted in an effective control of the larvae of M. ochroloma. In relation to the adult insects, only the extracts of tobacco powder and DalNeem showed effective control.

Key words: vegetable extracts, insecticidal plants, Melia azedarach

\section{Introdução}

O uso freqüente e indiscriminado de produtos químicos, para o controle de insetos-praga, muitas vezes acarreta a presença de altos níveis de resíduos tóxicos nos alimentos, desequilíbrio biológico, contaminações ambientais, intoxicações de seres humanos e outros animais, ressurgência de pragas, surtos de pragas secundárias e linhagens de insetos resistentes (Saxena, 1989). Uma alternativa para atenuar esses problemas é a utilização de aleloquímicos extraídos de plantas.

$\mathrm{Na}$ atualidade, as principais plantas das quais foram obtidas substâncias com atividade inseticida pertencem aos gêneros Nicotiana (Solanaceae), produtoras de nicotina e nornicotina; Derris, Lonchocarpus, Tephrosia e Mundulea (Fabaceae), produtoras de rotenóides; Chrysanthemum (Asteraceae), produtoras de piretrinas e Azadirachta (Meliaceae), produtoras de azadiractina (Vieira e Fernandes, 1999, apud Lovatto et al., 2004).

Dentre as famílias botânicas, Meliaceae vem se destacando como uma das mais importantes, tanto pelo número de espécies com atividade inseticida como pela eficiência dos seus extratos, especialmente em insetos mastigadores como lepidópteros e coleópteros (Vendramim, 1997). Nesses, podem causar repelência, alterar o crescimento, prolongar o desenvolvimento, impedir a muda, afetar a reprodução e causar mortalidade (Martinez, 2002). Uma das principais espécies é o cinamomo (Melia azedarach L.) que apresenta compostos limonóides também presentes no nim (Azadirachta indica A. Juss), espécie de origem asiática de uso difundido mundialmente com vistas ao controle de insetos.

Os extratos vegetais com atividade inseticida representam uma alternativa importante de controle de insetos-praga em pequenas áreas de cultivo, como as hortas, situação na qual a produção de extratos torna-se viável.

Microtheca ochroloma Stal pertence à ordem Coleoptera, família Chrysomelidae, sendo praga de brassicáceas nas fases larval e adulta, com maior infestação encontrada em couve-chinesa (Brassica chinensis L.). Ataque intenso pode resultar na inviabilização total da comercialização dessa olerícola, o que vem ocorrendo com frequiência por produtores orgânicos da região de Santa Maria, RS. Durante um cultivo de couve-chinesa (80 a 90 dias) podem ocorrer até quatro gerações de $M$. ochroloma (Marquini et al., 2003).

Em coleópteros crisomelídeos, a atividade de extratos de plantas inseticidas foi testada, basicamente, sobre Diabrotica speciosa (Germar). Carvalho e Castro (1987) referem-se a porcentuais de mortalidade de $89 \%$ e $97,5 \%$ obtidos, respectivamente, através do uso de extrato de folhas e de frutos de M. azedarach. O extrato de folhas reduziu o consumo em $8 \%$ e o extrato de frutos suprimiu totalmente o consumo alimentar dos insetos, em laboratório. Extratos de folhas, de caule, de flores e de frutos de M. azedarach foram avaliados por Ventura e Ito (2000) sendo que os extratos de flores e de frutos mostraram-se mais eficientes no controle de D. speciosa. Em ensaio realizado em estufa plástica, Seffrin (2006) comprovou que extratos aquosos a 10\% de frutos verdes e de ramos foram mais eficientes que extratos de casca e de folhas de M. azedarach e do produto comercial NIM-I-GO (à base de nim) no controle de D. speciosa.

Para Diabrotica virgifera (LeConte), Xie et al. (1991) demonstraram, a partir de estudos em laboratório, que a azadiractina foi tóxica para as larvas.

Fazolin et al. (1977) avaliaram o consumo médio da área foliar de plantas de feijão pelo crisomelídeo $\mathrm{Ce}$ - 
rotoma tingomarianus (Bechyné) na presença de nim, cinamomo pó e cinamomo semente e concluíram que, no tratamento com cinamomo pó, os insetos apresentaram menor consumo médio foliar.

Lovatto et al. (2004) referem-se à utilização de solanáceas como controladoras de insetos, por apresentarem metabólitos secundários com significativa toxicidade. Dentre estas, Nicotiana tabacum L., podendo ser utilizada contra vários insetos.

A necessidade de se propor alternativas de controle de $M$. ochroloma a produtores orgânicos da região de Santa Maria, RS e diante da inexistência de trabalhos sobre a eficiência de extratos de plantas inseticidas no controle desse inseto, realizou-se a presente pesquisa, em condições de laboratório. Além de meliáceas ( $M$. azedarach e $A$. indica) e solanáceas (N. tabacum e Capsicum frutescens L.) referidas na literatura como potencialmente eficientes, foram testadas também espécies de mirtáceas (Eugenia uniflora L., Syzygium cuminii (L.) Skeels e Eucalyptus sp.).

\section{Material e Métodos}

\section{Coleta dos insetos}

Os insetos adultos de M. ochroloma foram coletados em cultivos de couve-chinesa, no distrito de Arroio Grande (Latitude S 29³9'19,3'; Longitude W 53³9'05,9”), município de Santa Maria, RS, nos meses de junho e setembro de 2005 e agosto de 2006. Após, foram transportados ao Laboratório de Entomologia do Departamento de Defesa Fitossanitária do Centro de Ciências Rurais da Universidade Federal de Santa Maria (UFSM).

\section{Técnicas de criação dos insetos}

No Laboratório, os insetos adultos foram criados sob condição ambiente, em cubas de vidro $(0,79 \mathrm{~m}$ de diâmetro x $0,39 \mathrm{~m}$ altura), e alimentados com couvechinesa. A criação permitiu a obtenção de larvas com idade conhecida, que foram utilizadas nos ensaios.

\section{Extratos vegetais e tratamentos}

Todos os extratos utilizados foram aquosos, na concentração de $10 \% \mathrm{p} / \mathrm{v}$ e preparados seguindo a metodologia proposta por Vendramim e Castiglioni (2000).

Para o ensaio com larvas, foram utilizados extratos de folha de cinamomo, de ramo de cinamomo e de pó-de-fumo. As folhas e os ramos de cinamomo foram coletados em plantas situadas no Campus da UFSM, em setembro de 2005. O pó-de-fumo consiste do resíduo do processamento de folhas de fumo, após a moagem das mesmas, obtido em indústrias fumageiras da região de Santa Maria.

No primeiro ensaio de adultos, foram utilizados os extratos anteriormente citados e mais o extrato de DalNeem, também a $10 \%$. O DalNeem é um produto comercial, à base de frutos maduros de nim, com aproximadamente $3.000 \mathrm{ppm}$ de principio ativo e sob a forma de óleo emulsionável. A coleta das folhas e dos ramos de cinamomo, para esse ensaio, foi em abril de 2005, no Campus da UFSM.

No segundo experimento de adultos, foram testados extratos de frutos de pimenta malagueta (C. frutescens), folhas de pitangueira (E. uniflora), folhas de jambolão (S. cuminii) e folhas de eucalipto (Eucalyptus sp.). Esse material foi coletado em junho de 2006, no município de Santa Maria.

Em todos os ensaios foi utilizado um tratamento à base de água, como testemunha.

\section{Bioensaios}

Duas larvas, com três dias de idade, foram colocadas em tubos de vidro $(0,25 \mathrm{~m}$ de diâmetro $\mathrm{x} 0,85 \mathrm{~m}$ altura) contendo $12 \mathrm{~cm}^{2}$ de folha de couve-chinesa. As folhas foram previamente submergidas nos tratamentos por 20 segundos e, após, mantidas sobre papel toalha em condição ambiente, para evaporação do excesso de líquido. Os tubos foram vedados com algodão, mantido úmido, para evitar a saída dos insetos e conservados em câmara incubadora, à temperatura de $25^{\circ} \mathrm{C}$, umidade relativa de $70 \%$ e fotoperíodo de 14 horas. 
Para os insetos adultos, o mesmo procedimento adotado para o teste com as larvas foi repetido em dois ensaios, sendo colocados dois indivíduos por tubo. Os adultos utilizados nos experimentos foram trazidos diretamente do campo

Os ensaios constaram de 10 insetos por tratamento, com cinco repetições no primeiro ensaio com insetos adultos e com seis repetições nos demais. Foram feitas observações diárias, até o quinto dia, a fim de avaliar a mortalidade dos insetos.

Os resultados obtidos foram submetidos à análise de variância com as médias comparadas pelo teste de Tukey ao nível de significância de 5\%.

\section{Resultados e Discussão}

Verificou-se que a média da mortalidade diária acumulada das larvas, no primeiro dia, foi maior no tratamento com pó-de-fumo (Tabela 1) A partir do segundo dia, a mortalidade nos tratamentos de extratos de pó-de-fumo, de ramo e de folha de cinamomo não diferiram entre si, porém diferiram significativamente da testemunha. Já no primeiro dia houve mortalidade de mais de $50 \%$ das larvas expostas ao tratamento com pó-de-fumo, sendo que $100 \%$ de mortalidade foi atingida no quinto dia após a exposição. Também no quinto dia, insetos expostos a extratos de ramo e de folha de cinamomo atingiram mortalidade de $100 \%$ e $98 \%$, respectivamente. A maior mortalidade causada às larvas pelo pó-de-fumo no primeiro dia ocorreu em função do fumo conter nicotina, que apresenta ação de choque (contato e ingestão); já os compostos presentes no cinamomo, incluindo limonóides, apresentam efeito de ingestão e muitas vezes atuam na muda do inseto, o que pode ter resultado numa maior mortalidade de larvas no final do período de exposição.

No primeiro ensaio com insetos adultos (Tabela 2), a média da mortalidade diária acumulada dos insetos, no primeiro dia, foi maior no tratamento com DalNeem, não diferindo significativamente do extrato de pó-de-fumo e folha de cinamomo. A partir do segundo dia, a mortalidade nos tratamentos de DalNeem e póde-fumo não diferiram entre si, porém diferiram dos demais tratamentos, ocorrendo mortalidade de $82 \%$ e $74 \%$ dos insetos, respectivamente, no quinto dia após a exposição.

No segundo ensaio (Tabela 3), verificou-se que a mortalidade média diária acumulada dos insetos em todos os tratamentos não apresentou diferença estatisticamente significativa entre si. A mortalidade máxima observada foi de $17 \%$ dos insetos expostos a extrato de jambolão, no quinto dia após a exposição dos insetos aos diferentes tratamentos.

TABELA 1: Médias da mortalidade diária acumulada ( \pm Erro Padrão) de larvas de Microtheca ochroloma, alimentadas com folhas de couve-chinesa (Brassica chinensis) tratadas com extratos aquosos (na concentração de 10\%) de pó-de-fumo (Nicotiana tabacum), ramo e folha de cinamomo (Melia azedarach); além da testemunha (água destilada). Temperatura: $27 \pm 1^{\circ} \mathrm{C}$, UR $70 \pm 10 \%$, fotofase: $14 \mathrm{~h}$.

\begin{tabular}{lcccccc}
\hline & & \multicolumn{5}{c}{ Mortalidade diária acumulada (\%) $^{\mathbf{1}}$} \\
\cline { 2 - 7 } \multicolumn{1}{c}{ Tratamentos } & $\begin{array}{c}\text { No de } \\
\text { larvas }\end{array}$ & $\mathbf{1}$ & $\mathbf{5}$ & $\mathbf{3}$ & $\mathbf{4}$ & $\mathbf{5}$ \\
\hline Pó-de-fumo & 60 & $58,33 \pm 7,03 \mathrm{a}$ & $71,67 \pm 5,43 \mathrm{a}$ & $93,33 \pm 2,11 \mathrm{a}$ & $96,67 \pm 2,11 \mathrm{a}$ & $100,00 \pm 0,00 \mathrm{a}$ \\
\hline Ramo de cinamomo & 60 & $26,67 \pm 9,19 \mathrm{~b}$ & $51,67 \pm 11,67 \mathrm{a}$ & $86,67 \pm 7,60 \mathrm{a}$ & $88,33 \pm 7,92 \mathrm{a}$ & $100,00 \pm 0,00 \mathrm{a}$ \\
Folha de cinamomo & 60 & $13,33 \pm 3,33 \mathrm{~b}$ & $43,33 \pm 2,11 \mathrm{a}$ & $73,33 \pm 6,67 \mathrm{a}$ & $83,33 \pm 4,94 \mathrm{a}$ & $98,33 \pm 1,67 \mathrm{a}$ \\
\hline Testemunha & 60 & $0,00 \pm 0,00 \mathrm{c}$ & $1,67 \pm 1,67 \mathrm{~b}$ & $13,33 \pm 7,15 \mathrm{~b}$ & $21,67 \pm 10,46 \mathrm{~b}$ & $25,00 \pm 9,22 \mathrm{~b}$ \\
\hline CV $(\%)$ & & 43,33 & 31,43 & 23,67 & 24,21 & 12,12 \\
\hline
\end{tabular}

${ }^{1}$ Médias seguidas da mesma letra, nas colunas, não diferem pelo teste de Tukey $(\mathrm{P} \leq 0,5)$. 
TABELA 2: Médias da mortalidade diária acumulada ( \pm Erro Padrão) de adultos de Microtheca ochroloma, alimentados com folhas de couve-chinesa (Brassica chinensis) tratadas com extratos aquosos $(10 \%$ p/v) de pó-de-fumo (Nicotiana tabacum), ramo e folha de cinamomo (Melia azedarach), DalNeem (produto comercial à base de Azadirachta indica) e água (testemunha). Temperatura: $27 \pm 1{ }^{\circ} \mathrm{C}, \mathrm{UR}$ $70 \pm 10 \%$, fotofase: $14 \mathrm{~h}$.

\begin{tabular}{lcccccc}
\hline & & \multicolumn{5}{c}{ Mortalidade diária acumulada (\%) $^{\mathbf{1}}$} \\
\cline { 2 - 7 } \multicolumn{1}{c}{ Tratamentos } & $\begin{array}{c}\text { No de } \\
\text { adultos }\end{array}$ & $\mathbf{1}$ & $\mathbf{2}$ & $\mathbf{3}$ & $\mathbf{4}$ & $\mathbf{5}$ \\
\hline DalNeem & 50 & $40,00 \pm 17,61 \mathrm{ab}$ & $70,00 \pm 12,25 \mathrm{a}$ & $76,00 \pm 12,49 \mathrm{a}$ & $78,00 \pm 13,19 \mathrm{a}$ & $82,00 \pm 13,56 \mathrm{a}$ \\
\hline $\begin{array}{l}\text { Pó-de-fumo } \\
\begin{array}{l}\text { Folha de } \\
\text { cinamomo }\end{array}\end{array}$ & 50 & $38,00 \pm 15,94 \mathrm{a}$ & $54,00 \pm 16,31 \mathrm{a}$ & $66,00 \pm 12,88 \mathrm{a}$ & $70,00 \pm 12,25 \mathrm{a}$ & $74,00 \pm 13,27 \mathrm{a}$ \\
$\begin{array}{l}\text { Ramo de } \\
\text { cinamomo }\end{array}$ & 50 & $2,00 \pm 2,00 \mathrm{ab}$ & $2,00 \pm 2,00 \mathrm{~b}$ & $4,00 \pm 4,00 \mathrm{~b}$ & $6,00 \pm 6,00 \mathrm{~b}$ & $6,00 \pm 6,00 \mathrm{~b}$ \\
$\begin{array}{l}\text { Testemunha } \\
\text { CV (\%) }\end{array}$ & 50 & $0,00 \pm 0,00 \mathrm{~b}$ & $2,00 \pm 2,00 \mathrm{~b}$ & $6,00 \pm 4,00 \mathrm{~b}$ & $8,00 \pm 3,74 \mathrm{~b}$ & $10,00 \pm 3,16 \mathrm{~b}$ \\
\hline
\end{tabular}

${ }^{1}$ Médias seguidas da mesma letra, nas colunas, não diferem pelo teste de Tukey $(\mathrm{P} \leq 0,5)$.

TABELA 3: Médias da mortalidade diária acumulada ( \pm Erro Padrão) de adultos de Microtheca ochroloma, alimentados com folhas de couve-chinesa (Brassica chinensis), tratados com extratos aquosos $(10 \%$ $\mathrm{p} / \mathrm{v}$ ) de fruto de pimenta-malagueta (Capsicum frutescens), folha de pitangueira (Eugenia uniflora), folha de jambolão (Syzygium cuminii), folha de eucalipto (Eucalyptus sp.) e água (testemunha). Temperatura: $27 \pm 1^{\circ} \mathrm{C}$, UR $70 \pm 10 \%$, fotofase: $14 \mathrm{~h}$.

\begin{tabular}{lcccccc}
\hline & & \multicolumn{5}{c}{ Mortalidade diária acumulada (\%) $^{\mathbf{1}}$} \\
\cline { 2 - 7 } Tratamentos & $\begin{array}{c}\text { No de } \\
\text { adultos }\end{array}$ & $\mathbf{1}$ & $\mathbf{2}$ & $\mathbf{3}$ & $\mathbf{4}$ & $\mathbf{5}$ \\
\hline Pimenta-malagueta & 60 & $3,33 \pm 3,33 \mathrm{a}$ & $5,00 \pm 5,00 \mathrm{a}$ & $6,67 \pm 4,94$ a & $6,67 \pm 4,94 \mathrm{a}$ & $8,33 \pm 6,54 \mathrm{a}$ \\
Pitangueira & 60 & $1,67 \pm 1,67 \mathrm{a}$ & $1,67 \pm 1,67 \mathrm{a}$ & $5,00 \pm 3,42 \mathrm{a}$ & $6,67 \pm 3,33 \mathrm{a}$ & $6,67 \pm 3,33 \mathrm{a}$ \\
Jambolão & 60 & $3,33 \pm 2,11 \mathrm{a}$ & $6,67 \pm 3,33 \mathrm{a}$ & $8,33 \pm 4,01$ a & $11,67 \pm 6,54 \mathrm{a}$ & $16,67 \pm 7,6 \mathrm{a}$ \\
Eucalipto & 60 & $0,00 \pm 0,00 \mathrm{a}$ & $1,67 \pm 1,67 \mathrm{a}$ & $5,00 \pm 3,42 \mathrm{a}$ & $5,00 \pm 3,42 \mathrm{a}$ & $10,00 \pm 5,16 \mathrm{a}$ \\
\hline Testemunha & 60 & $0,00 \pm 0,00 \mathrm{a}$ & $3,33 \pm 2,11 \mathrm{a}$ & $3,33 \pm 2,11 \mathrm{a}$ & $5,00 \pm 3,42 \mathrm{a}$ & $8,33 \pm 5,43 \mathrm{a}$ \\
\hline CV (\%) & & 79,61 & 79,61 & 79,61 & 79,61 & 79,61 \\
\hline
\end{tabular}

${ }^{1}$ Médias seguidas da mesma letra, nas colunas, não diferem pelo teste de Tukey $(\mathrm{P} \leq 0,5)$.

Os resultados obtidos são inferiores aos encontrados por Carvalho e Castro (1987), para adultos de $D$. speciosa, que citam um percentual de mortalidade de $89 \%$ através do uso de extrato de folhas de $M$. azedarach, enquanto que para M. ochroloma esse porcentual não foi superior a $6 \%$. Também diferem dos obtidos por
Seffrin (2006) que comprovou a eficiência de extrato de ramos de cinamomo no controle de insetos adultos de $D$. speciosa, em estufa plástica, quando comparado, dentre outros, a produto comercial à base de nim e a extrato de folha de cinamomo. 
Portanto, a partir dos resultados obtidos, pode-se concluir que, para o controle de larvas de M. ochrolo$m a$, extratos aquosos a $10 \%$ de pó-fumo, ramo e folhas de cinamomo são eficientes, ocasionando mortalidade superior a $80 \%$ a partir do terceiro dia de exposição dos insetos ao pó-de-fumo e ao ramo de cinamomo e do quarto dia ao de folha de cinamomo. Já para os insetos adultos, a maior eficiência é obtida através da utilização de extratos de DalNeem e pó-de-fumo, que causam mortalidade superior a $80 \%$ a partir do quinto dia da exposição ao DalNeem e próximo e esse valor (74\%) para o pó-de-fumo na mesma data. Porém, deve ser comprovada tanto a eficiência dos extratos no campo quanto a ausência de toxicidade às populações de inimigos naturais de M. ochroloma, antes de serem recomendados a produtores que se dedicam ao cultivo de brassicáceas, plantas hospedeiras deste crisomelídeo.

\section{Referências}

Carvalho, S. M.; Castro, B. R. R. 1987. Efeito de plantas tóxicas no controle da vaquinha Diabrotica speciosa Germar em laboratório. Anais da II Reunião Nacional de Pesquisa de Feijão, Goiânia, Brasil, p.49.

Fazolin, M.; Estrela, J. L. V.; Argolo, V. M. 1977. Seleção de substâncias no controle da vaquinha do feijoeiro Cerotoma tingomarianus Bechyné (Coleoptera, Chrysomelidae). EMBRAPA/Centro Nacional de Pesquisa Agroflorestal do Acre, Rio Branco, Brasil, 5pp. (Boletim de Pesquisa).
Lovatto, P. B.; Goetze, M.; Thomé, G. C. H. 2004. Efeito de extratos de plantas silvestres da família Solanaceae sobre o controle de Brevicoryne brassicae em couve (Brassica oleraceae var. acephala). Ciência Rural, 34 (4): 971-978.

Marquini, F.; Picanço, M. C.; Moura, M. F.; Oliveira, I. R. 2003. Ciclo de vida de Microtheca ochroloma Stal, 1860 (Coleoptera, Chrysomelidae, Chrysomelinae). Revista Ceres, 50: 283-291.

Martinez, S. S. 2002. Outras Meliáceas. In: Martinez, S. S. (Ed.). O Nim Azadirachta indica - natureza, usos múltiplos, produção. IAPAR, Londrina, Brasil, p.111-112.

Saxena, R. 1989. Insecticides from Neem. In: Arnason, J. T.; Philogene B. J. R. \& Morand, P. (eds). Insecticides of plant origin. ACS, Washington, USA, p.110-129.

Seffrin, R. C. A. S. 2006. Bioatividade de extratos vegetais sobre Diabrotica speciosa (Germar, 1824) (Coleoptera, Chrysomelidae). Tese de Doutorado, Universidade Federal de Santa Maria, Brasil, 89pp.

Vendramim, J. D. 1997. Uso de plantas inseticidas no controle de pragas. II Ciclo de Palestras sobre Agricultura Orgânica, Fundação Cargill, São Paulo, Brasil, p.64-69.

Vendramim, J. D.; Castiglioni, E. 2000. Aleloquímicos, resistência de plantas e plantas inseticidas. In: Guedes, J. C.; Costa, I. D. \& Castiglioni, E. (Org.). Bases e Técnicas do Manejo de Insetos. Pallotti, Santa Maria, Brasil, p.113-128.

Ventura, M. U.; Ito, M. 2000. Antifeedant activity of Melia azedarach (L.) extracts to Diabrotica speciosa (Genn.) (Coleoptera: Chrysomelidae) beetles. Brazilian Archives of Biology and Technology, 43 (2): 215-219.

Xie, Y. S.; Gagnon, D.; Arnason, J. T.; Philogène, B. J. R.; Lambert, J. D. H.; Kaminski, J.; Morand, P.; Timmins, G.; Werstiuk, N. H. 1991. Effects of azadirachtin on the western corn rootworm, Diabrotica virgifera virgifera (LeConte) (Coleoptera: Chrysomelidae). Canadian Entomologist, 123 (3): 707-710. 